\title{
Physiological response and transcriptome analysis of Prunus mume to early salt stress
}

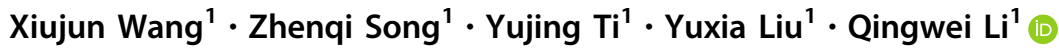

Received: 5 December 2020 / Accepted: 29 April 2021 / Published online: 15 May 2021

(C) The Author(s) 2021

\begin{abstract}
Soil salinisation is an important factor limiting the cultivation and distribution of Prunus mume. Therefore, identifying the regulation mechanism of salt tolerance of $P$. mume will promote its cultivation and molecular breeding. In this study, the garden tree species $P$. mume 'Meiren' was investigated, with a soil $\mathrm{NaCl}$ content (w/w) of $0.3 \%$. Photosynthetic gas exchange parameters, relative electrical conductivity, malondialdehyde, osmoregulation substances, and antioxidant enzyme activity were measured in the early (3d), middle (10d), and late (30d) stages of stress. Salt treatment was applied for $0 \mathrm{~h}, 6 \mathrm{~h}, 24 \mathrm{~h}$, and $72 \mathrm{~h}$; following this, the gene library was constructed from the leaves. The results showed that the CBL-CIPK, mitogen-activated protein kinase, calcium-dependent protein kinase, and abscisic acid signalling pathways were involved in salt stress signal transduction of $P$. mume. Furthermore, bHLH, WRPK, ERF, and MYB were identified as potential key transcription factor families of salt tolerance in $P$. mume. Combined with determined physiological responses, the results showed that early salt stress inhibited the activities of RuBisCO, chlorophyll synthase, glutamyl tRNA reductase, divinyl reductase, and magnesium chelatase; resultantly, this led to a reduction in the photosynthetic rate. Also, it was found that P5CS, SS, LEA, and dehydrin regulated the synthesis of proline, soluble sugar, and macromolecular protein to alleviate osmotic stress. POD gene plays an important role in scavenging reactive oxygen species due to its regulation of POD activity. The findings of this research reveal the internal mechanism of the physiological response and provide a foundation for the construction of a genetic regulatory network in response to salt stress in $P$. mume.
\end{abstract}

Keywords Prunus mume $\cdot$ Transcriptome $\cdot$ Signal transduction $\cdot$ Salt tolerance $\cdot$ QRT-PRC

\section{Abbreviations}

ABA

AKT

ALDH

AQP

bHLH

bZIP

Abscisic acid

Potassium channel

Aldehyde dehydrogenase

Aquaporin

CAMTA

Basic Helix-Loop-Helix

Basic leucine zipper

Calmodulin-binding transcription activator

Supplementary Information The online version contains supplementary material available at https://doi.org/10.1007/ s13562-021-00680-2.

Qingwei Li

lqw6809@bjfu.edu.cn

1 Beijing Key Laboratory of Ornamental Plants Germplasm Innovation and Molecular Breeding, National Engineering Research Center for Floriculture, Beijing, Laboratory of Urban and Rural Ecological Environment, School of Landscape Architecture, Beijing Forestry University, Beijing, China

$\begin{array}{ll}\text { CBL } & \text { Calcineurin B-like protein } \\ \text { CBF } & \text { Core binding factor } \\ \text { CDPK } & \text { Calcium-dependent protein kinase } \\ \text { CHX } & \text { Cation/ }{ }^{+} \text {exchangers } \\ \text { Ci } & \text { Intercellular } \mathrm{CO}_{2} \text { concentration } \\ \text { CIPK } & \text { CBL-interacting protein kinase } \\ \text { CNGCs } & \text { Cyclic-micleotide gated channels } \\ \text { DEGs } & \text { Differentially expressed genes } \\ \text { DHN } & \text { Dehydrin } \\ \text { ERF } & \text { ETS2 Repressor Factor } \\ \text { Gs } & \text { Stomatal conductivity } \\ \text { GTLs } & \text { GT element-binding-like proteins } \\ \text { HSP } & \text { Heat shock protein } \\ \text { LEA } & \text { Late embryogenesis abundant protein } \\ \text { MAPK } & \text { Mitogen-activated protein kinase } \\ \text { MDA } & \text { Malonic dialdehyde } \\ \text { MT } & \text { Metallothionein protein } \\ \text { MYB } & \text { Myeloblastosis } \\ \text { MYC } & \text { MYC proto-oncogene, bHLH transcription } \\ & \text { factor }\end{array}$

CBL Calcineurin B-like protein

CDPK Calcium-dependent protein kinase

$\mathrm{CHX} \quad \mathrm{Cation} / \mathrm{H}^{+}$exchangers

CIPK CBL-interacting protein kinase

CNGCs Cyclic-micleotide gated channels

DEGs Differentially expressed genes

DHN

Dehydrin

ERF

Stomatal conductivity

GTLs

HSP

GT element-binding-like proteins

LEA Late embryogenesis abundant protein

MAPK Mitogen-activated protein kinase

MDA

MT

Malonic dialdehyde

MYB

factor 


$\begin{array}{ll}\text { NHX } & \text { Sodium/hydrogen exchanger } \\ \text { NSCCs } & \text { Nonselective cation channels } \\ \text { P5CS } & \text { Pyrroline-5-carboxylate synthase } \\ \text { PLD } & \text { Phospholipase D } \\ \text { Pn } & \text { Net photosynthetic rate } \\ \text { POD } & \text { Peroxidase } \\ \text { PP2C } & \text { Protein phosphatase 2C } \\ \text { Pro } & \text { Free proline } \\ \text { PrxR } & \text { Peroxiredoxin } \\ \text { PS I } & \text { Photosystem I reaction centre } \\ \text { PYL } & \text { Pyrrolysine-tRNA } \\ \text { PYR } & \text { Pyramus } \\ \text { Rubisco } & \text { Ribulose-1,5-bisphosphate Carboxylase/ } \\ & \text { oxygenase activase } \\ \text { SnRK } & \text { Serine/threonine-protein kinase } \\ \text { SOD } & \text { Surperoside dismutase } \\ \text { SS } & \text { Soluble sugar } \\ \text { SUSY } & \text { Sucrase synthase } \\ \text { TF } & \text { Transcription factor } \\ \text { Tr } & \text { Transpiration rate }\end{array}$

\section{Introduction}

The salinisation of soil has become a major global resource concern and environmental issue. The salt content in soil affects the geographical distribution of plants in nature, whilst also limiting the productivity of plants (Zhu 2016; Agarwal et al. 2020). Increased concentrations of $\mathrm{Na}^{+}$and $\mathrm{Cl}^{-}$affect plant metabolism and reduce the rate of photosynthesis, and consequently, slow down plant growth (Waqas et al. 2021; Zhao et al. 2020). In the process of long-term evolution and environmental adaptation, plants have formed a series of physiological and molecular mechanisms in response to salt stress, which can be divided into two categories: the first is salt tolerance, whereby effector molecules directly participate in changes to the physiological cellular metabolism, and therefore, contribute to salt tolerance, and the second is regulatory molecules, which are located upstream of effector molecules and are involved in signal transduction, primarily including various kinases and transcription factors comprising the signal transduction system (Chen et al. 2021).

Mei is a traditional, well-known flower in China, which has been cultivated for over 3000 years (Zhang et al. 2012). Soil salinity and alkali content are important factors that can limit the distribution of P. mume (Yang et al. 2018; Li 2009). The role of core binding factor (CBF) (NF-Y), phospholipase D (PLD), late embryogenesis abundant protein (LEA), basic leucine zipper (bZIP), and small heat shock proteins (HSP) has been established in abiotic stress (Du et al. 2012; Yang et al. 2016; Peng et al. 2016; Wan et al. 2016). Overexpression of PmLEA can improve cold and drought resistance of tobacco (Bao et al. 2017). Moreover, $\mathrm{CBF}$ is also associated with cold resistance, salt tolerance, and antioxidant capacity of $P$. mume (Yang et al. 2016). However, the salt tolerance mechanism of $P$. mume has yet to be systematically studied, and the signal transduction pathway involved in salt tolerance remains unclear. To expose the mechanism of salt tolerance of $P$. mume and screen the key genes of salt tolerance, we aim to provide a theoretical basis for the breeding of new salt tolerant varieties and to promote the cultivation and distribution of $P$. mume in saline alkali soil.

\section{Materials and methods}

\section{Plant material and salt treatment}

The experimental aspect of this study was carried out in the nursery greenhouse of Beijing Forestry University $\left(116.3^{\circ} \mathrm{E}, 40.0^{\circ} \mathrm{N}\right)$, between June-August. The annual branches of $P$. mume 'Meiren' were collected from one mother plant and grafted onto the same growing rootstock of $P$. mume 'Meiren'. Seedlings with relatively uniform growth were planted in plastic flowerpots with a size of $35 \mathrm{~cm} \times 30 \mathrm{~cm} \times 35 \mathrm{~cm}$ in March, with trays arranged underneath the flowerpots; the planting density was 1 plant/ pot. The culture medium was mixed by volume ratio of peat soil: perlite: river sand $=2: 1: 1$, and the mass of each pot was $7.0 \mathrm{~kg}$.

When each test seedling entered the vigorous growth period, the pot soil was controlled for 7 days to ensure the soil was dry and to facilitate the even distribution of salt during salt application. Once the pot soil was dry, $1500 \mathrm{ml}$ of distilled water was poured into the control pots, whilst $1500 \mathrm{ml}$ of salt solution was poured into the test pots, and the exuded water from the pots was poured into the pots again. The soil $\mathrm{NaCl}$ content $(\mathrm{w} / \mathrm{W}$ ) of control group and experimental group were $0.0 \%$ and $0.3 \%$ respectively, 3 replicates of each treatment were conducted. The soil moisture content was maintained at $60-80 \%$ by adding clean water every 5 days. During treatment $0 d, 3 d, 10 d$, and 30d, samples were taken in the morning for index determination, and 2 or 3 new middle and upper functional leaves were taken from different directions of each plant. At $0 \mathrm{~h}, 6 \mathrm{~h}, 24 \mathrm{~h}$, and $72 \mathrm{~h}$ of salt treatment, new middle and upper functional leaves were taken for transcriptome sequencing and gene expression. The transcripts were marked as T0, T6, T24, and T72, and each treatment was 
repeated 3 times, resulting in a total of 12 samples. The samples were immediately frozen in liquid nitrogen after collection and were stored at $-80{ }^{\circ} \mathrm{C}$ until use.

\section{Physiological index determination}

The direct leaching method was used to determine chlorophyll content, whilst the conductivity meter method was used to determine relative conductivity, and the determination of malondialdehyde (MDA) was achieved through the thiobarbituric acid method. The determination of soluble sugar was attained by the anthrone colorimetric method, acid ninhydrin colorimetry was used to determine free proline (Pro), and the Coomassie Brilliant Blue G250 staining method was used to determine soluble protein. In addition, the nitrogen blue tetrazolium (NBT) photoreduction method was implemented to determine superoxide dismutase (SOD) activity, and the guaiacol method was used to determine peroxidase (POD) activity (Li 2003).

The photosynthetic gas exchange parameters, such as net photosynthesis $(\mathrm{Pn})$, transpiration $(\mathrm{Tr})$, stomatal conductance $(\mathrm{Gs})$, and intercellular $\mathrm{CO}_{2}$ concentration $(\mathrm{Ci})$, were measured by LI-6400 portable photosynthetic apparatus. The temperature was maintained at $30{ }^{\circ} \mathrm{C}$, the light intensity was $1,000 \mu \mathrm{mol} \cdot \mathrm{m}^{-2} \cdot \mathrm{s}^{-1}$, and the $\mathrm{CO}_{2}$ concentration was $400 \mu \mathrm{mol} \cdot \mathrm{mol}^{-1}$.

\section{RNA extraction, library construction and data processing}

RNA was extracted from the plant samples using RNAprep Pure Plant Kit (Polysaccharides and Polyphenolics-rich). Agarose gel electrophoresis was used to determine the extent of RNA degradation and contamination, whilst Nanodrop was used to detect the purity of the extracted RNA (OD260/280 ratio). In addition, a qubit accurately quantified the RNA concentration, and Agilent 2100 accurately detected RNA integrity. The cDNA library was sequenced using Illumina HiSeq 4000.

The raw image data files obtained by high-throughput sequencing were converted into original sequenced reads by CASAVA base calling analysis; the raw reads were then filtered to obtain clean reads to be used as the basis of subsequent analysis. The data processing steps were as follows: 1) reads with adapters were removed; 2) reads containing $\mathrm{N}$ (undetermined bases) greater than $10 \%$ were removed; and 3) low quality reads were removed (Qphred $\leq 20$ bases that accounted for more than 50\% of the read length). HISAT software was selected to perform genomic localisation analysis on the filtered sequencing sequence. The raw reads were uploaded to NCBI, reference: PRJNA678761.

\section{Screening and enrichment analysis of differentially expressed Unigene}

RNA-seq expression quantification was based on the fragments per kilo base per million mapped reads (FPKM) values. Also, the negative binomial distribution model was used to calculate the hypothesis test probability (pvalue). The false discovery rate (FDR) was calculated to correct for multiple testing, as applicable for multi-hypothesis testing. In addition, the $p$ value adjusted (padj) $<0.05$ was used as a differential gene screening standard.

The Gene Ontology (http://www.geneontology.org/) was divided into three parts: molecular function, biological process, and cellular component (Young et al. 2010). Pathway enrichment analysis was based on the pathway in the Kyoto Encyclopedia of Genes and Genomes (KEGG) database. Also, the hypergeometric test was used to identify the pathways that were significantly enriched in the differentially expressed genes compared with the entire genome background (Kanehisa 2008). Furthermore, KOBAS (2.0) was used for pathway enrichment analysis.

\section{Transcript screening and statistics}

Plant transcription factor prediction was carried out using iTAK software. The basic principle of this process relied on the use of the TF family and rules defined in the database to identify transcription factors through hmmscan in the HMMER suite. The identification and classification of transcription factors correspond to the method of PerezRodriguez et al. (2010).

\section{qRT-PCR verification}

cDNA was synthesised using Primer Script ${ }^{\mathrm{TM}}$ RT Master Mix. PCR was then performed based on the CFX Connect Real-time System (Bio-Rad, USA) using SYBR Primix Ex TaqTM II (Tli RNaseH Plus). The $25 \mu \mathrm{L}$ reaction system contained $2 \mu \mathrm{L}$ of template, $1 \mu \mathrm{L}$ of upstream and downstream primers $(10 \mu \mathrm{M})$ (Table 1$), 8.5 \mu \mathrm{L}$ RNase Free $\mathrm{dH}_{2} \mathrm{O}$, and $12.5 \mu \mathrm{L}$ of SYBR Premix Ex TaqII (Takara). All experiments were performed as 3 biological replicates. The ubiquitin C (UBC) gene was used as the internal reference gene (Wang et al. 2014). The relative expression levels of the genes were calculated using the double standard curve method. 
Table 1 Primer sequences for quantitative expression

\begin{tabular}{lllll}
\hline Gene ID & Upstream primer sequence 5'-3' & $\begin{array}{l}\text { Downstream primer sequence 5'- } \\
3^{\prime}\end{array}$ & $\begin{array}{l}\text { Annealing temperature/ } \\
\text { Tm }\end{array}$ & $\begin{array}{l}\text { Amplification length/ } \\
\text { bp }\end{array}$ \\
\hline LOC103333990 & TAAGCGTTAGGGAGCGAGTC & AGAAAGGTGGCGATTGAGG & 55 & 473 \\
LOC103333005 & GTCCTAATGCCGTAAACCC & CTTCACTGCCAGCAAAATAA & 55 & 326 \\
LOC103344682 & GTTTCACAACTCTGACCCTT & GCATCATTCACTACTCCCTC & 55 & 148 \\
LOC103323454 & CTGCCATTTGATGACGCTA & CCAATCCTTGTTTTCTGCTT & 55 & 395 \\
LOC103323643 & GGTCCCCAAAATAAGCAAG & TGTAACCCTAAGTTCAAGCA & 55 & 262 \\
LOC103323814 & CGTTCCCAGATCCCTGTAT & GCACTTTGGTCCTTTCCAT & 55 & 321 \\
LOC103334364 & AGCAGCAAGCAGCACATAA & TTCAATCTCGTCCAAAGCA & 55 & 467 \\
LOC103328315 & ACTTCACTTTCTCCTTCGGTC & TGAGGTCCAGAGGGTCCAA & 55 & 284 \\
LOC103334065 & ATCAAAGGGTTGTTCTGCC & TGGGTCAAGTTAGTGGTGG & 55 & 126 \\
LOC103327836 & AGAGTAATGCTACCCGAAGA & CTGACAAGAAGGCAGAAAA & 55 & 113 \\
LOC103340164 & TCCTTGACATTGGACCCTG & CTAATTTCTGCCCCACCCT & 55 & 164 \\
LOC103340279 & CACGGATGACTCGTTGCTC & GTGCCTTGATGCCTTTGTA & 55 & 116 \\
AT5G53300 & GCAGGTGGAGTGTTCCTTGTGA & GGCAGGGCTCCACTGTTCTTT & 55 & 146 \\
\hline
\end{tabular}

\section{Results}

\section{Salt response: physiological characteristics}

The photosynthetic gas exchange parameters, relative conductivity, MDA level, osmotic regulators, and antioxidant enzyme activities were measured at early-stage stress (3d), mid-stage stress (10d), and late-stage stress (30d) (Fig. 1a-d). The results of $\mathrm{Pn}, \mathrm{Gs}$, and SOD enzyme showed a downward trend over time; contrastingly, relative conductivity, free Pro, and POD enzyme activities showed an upward trend. MDA, soluble sugar, soluble protein, and $\mathrm{Ci}$ showed an initial increasing trend, followed by decreasing levels. On the other hand, Tr initially decreased, and then increased, but the value remained lower poststress than pre-stress. Moreover, the physiological characteristics of $P$. mume in response to salt stress varied in different stress periods. In the early period of stress, the photosynthetic gas exchange parameters, relative conductivity, MDA, and soluble sugar content changed significantly $(P \leq 0.05)$; in the mid-stage of stress, the indicators all changed with the exception of $\mathrm{Ci}$, which returned to a normal value; in the late stage of stress, soluble sugar and $\mathrm{Ci}$ returned to normal levels, while $\mathrm{Pn}, \mathrm{Tr}$, and SOD showed values lower than those of control group, and relative conductivity, MDA, free proline, and soluble protein were higher than normal levels. Under salt stress, photosynthetic gas exchange, membrane system activity, osmotic adjustment substance, and antioxidant enzyme activity showed significant positive correlation (Fig. 1e).

The seedlings with relatively consistent growth were planted in individual flowerpots, and the $\mathrm{NaCl}$ content (w/ w) of the soil for the control and test plants was $0.0 \%$ and
$0.3 \%$, respectively. The samples were collected at $0 \mathrm{~d}, 3 \mathrm{~d}$, 10d, and 30d to determine each index. According to Duncan's test, different letters represent a different level of significance $(P \leq 0.05)$. The correlation heat map was drawn using GraphPad Prism 8, whereby A represents photosynthetic gas exchange parameters, B represents membrane system related indexes, $\mathrm{C}$ represents osmotic adjustment substances, and D represents antioxidant enzyme activity.

\section{Transcriptome sequencing analysis}

To investigate the expression patterns of the $P$. mume leaves at $0 \mathrm{~h}, 6 \mathrm{~h}, 24 \mathrm{~h}$, and $72 \mathrm{~h}$ of salt stress, four samples (T0, T6, T24, and T72) were transcribed using Illumina HiSeq 4,000. After removing the splice sequence, repetitive sequence, unknown reading segment, and lowquality reading segment, 66,150,124, 51,282,181, $54,613,020$, and 52,907,542 clean reads were obtained, which covered $85.53 \%, 84.57 \%, 83.05 \%$, and $83.59 \%$ of the genome sequence of $P$. mume, respectively (Table 2).

\section{Gene expression analysis}

A total of 64,916 transcripts were obtained from the 4 libraries constructed based on sequencing. The DEGs were filtered with expression levels $\log _{2}$ fold change $>1$ or $<-$ $1, q$ value $<0.005$ in each pairwise comparison, and 5,161 genes were differentially expressed under salt stress (T6 vs. T0, T24 vs. T0, T72 vs. T0). Among these three groups, the greatest number of differentially expressed genes was 3140 at T72. In T6 vs. T0, the differentially expressed genes included more down-regulated genes than up-regulated 


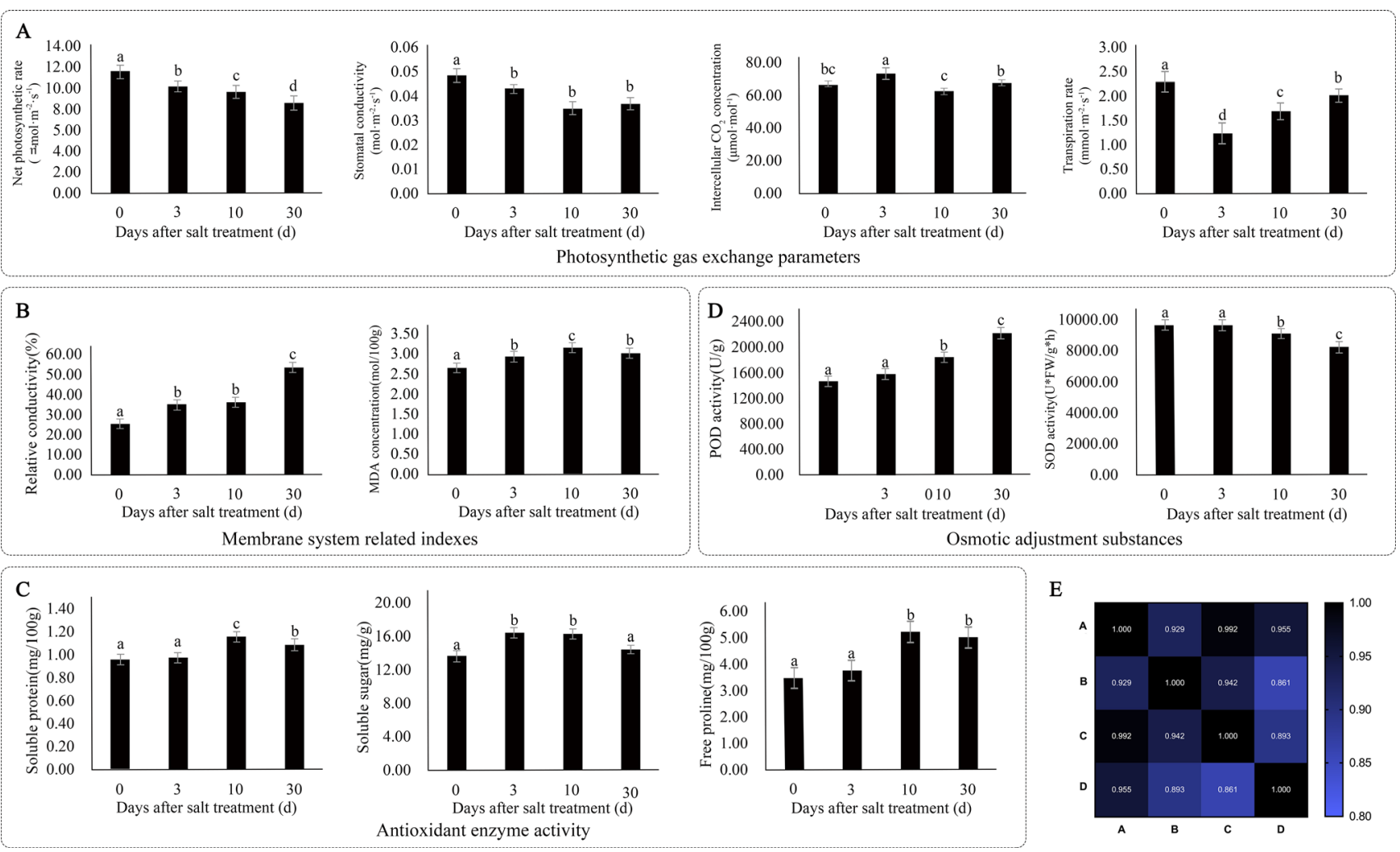

Fig.1 a Effects of salt stress on photosynthetic gas exchange parameters. b Effects of salt stress on malondialdehyde and relative conductivity. c Effects of salt stress on osmotic adjustment substances. d Effects of salt stress on antioxidant enzyme activity. e The correlation heat map of four physiological indexes: photosynthetic gas exchange parameters, membrane system related indexes, osmotic adjustment substances and antioxidant enzyme activity
Table 2 Statistical results of Illumina HiSeq 4000 sequencing

\begin{tabular}{llllllll}
\hline Sample & Original reading & Filtered reads & Filter data/G & Error rate/\% & Q30/\% & GC/\% & Coverage/\% \\
\hline T0 & $67,850,617$ & $66,150,124$ & 9.92 & 0.01 & 93.45 & 45.54 & 85.53 \\
T6 & $52,550,703$ & $51,282,181$ & 7.69 & 0.01 & 93.44 & 45.41 & 84.57 \\
T24 & $56,131,598$ & $54,613,020$ & 8.19 & 0.02 & 93.11 & 45.47 & 83.04 \\
T72 & $54,398,926$ & $52,907,542$ & 7.94 & 0.02 & 93.08 & 45.74 & 83.59 \\
\hline
\end{tabular}

genes, while at T24 vs. T0 and T72 vs. T0 the differentially expressed genes presented more up-regulation than downregulation (Fig. 2a). The differential expression of genes varied at different stress time points. Compared with $0 \mathrm{~h}$ stress, a total of 527 genes were significantly differentially expressed at $6 \mathrm{~h}, 24 \mathrm{~h}$, and $72 \mathrm{~h}$ salt stress. It is possible that these co-expressed genes are related to the salt response (Fig. 2b).

Hierarchical clustering based on gene expression patterns (Fig. 3a) identified T24 and T72 as being more likely to be clustered together. As the stress time increased, the gene expression patterns became closer. Hierarchical clustering was used to cluster the relative expression levels of differential genes $\log _{2}$ (ratios) (Fig. 3b). The two most significant enrichment groups were Subcluster 61,371 genes and Subcluster 31,092 genes. At $6 \mathrm{~h}$, a smaller fluctuation was identified than at $0 \mathrm{~h}$, which then increased or decreased significantly at $24 \mathrm{~h}$. T72 followed essentially the same pattern as $\mathrm{T} 24$, as the expression of a considerable number of genes changed significantly at $24 \mathrm{~h}$, and this trend of change was maintained at $72 \mathrm{~h}$ of stress.

Twelve genes were selected to verify the transcriptome sequencing results (Enclose Table 1), whereby strong positive correlation was determined between the results of RNA-seq and qRT-PCR (Fig. 4); the correlation coefficients $\mathrm{R} 2$ in $\mathrm{T} 6$ vs. T0, T24 vs. T0, and $\mathrm{T} 72$ vs. T0 were $0.89,0.90$, and 0.88 , respectively. This signified the reliability of the transcriptome sequencing data. 


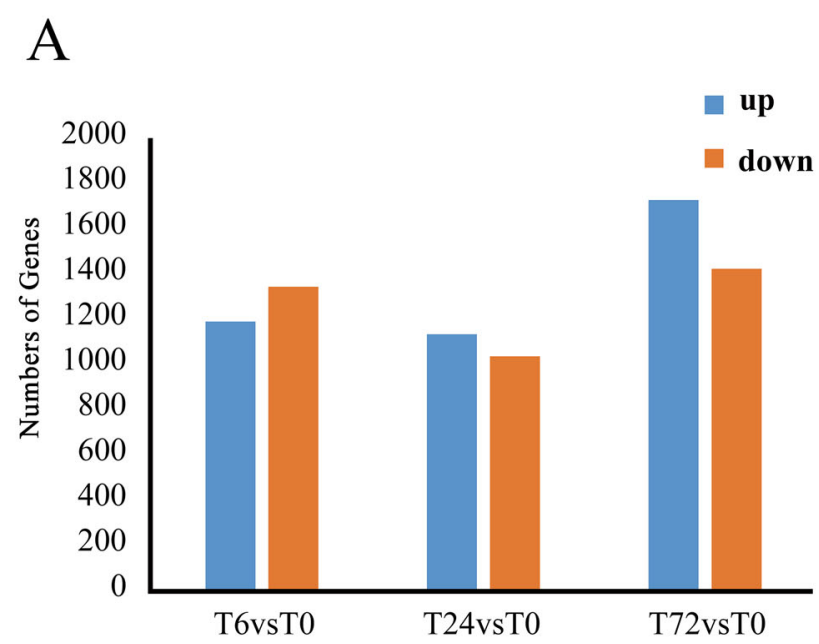

Fig. 2 a Histogram and b Venn diagram of differentially expressed genes. A total of 5161 genes were differentially expressed under salt stress; the histogram depicts the number of genes up-regulated or

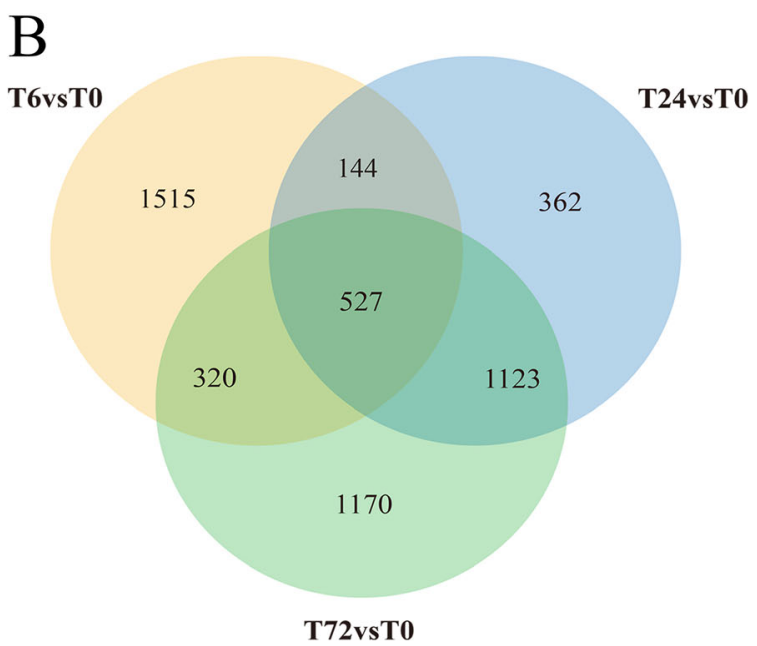

down-regulated in T6 vs. T0, T24 vs. T0, and T72 vs. T0. The Venn diagram shows the number of differentially expressed genes overlapping from the three time points after salt stress
A

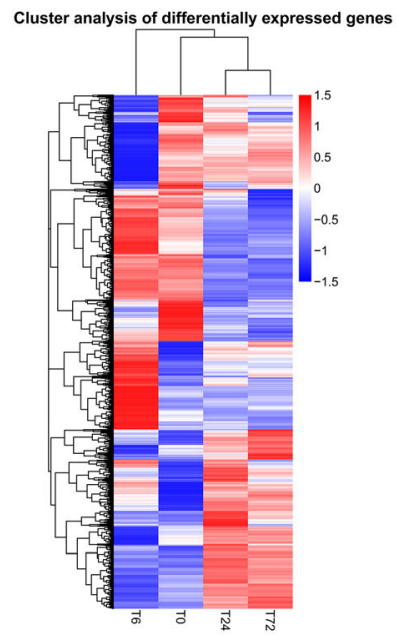

B
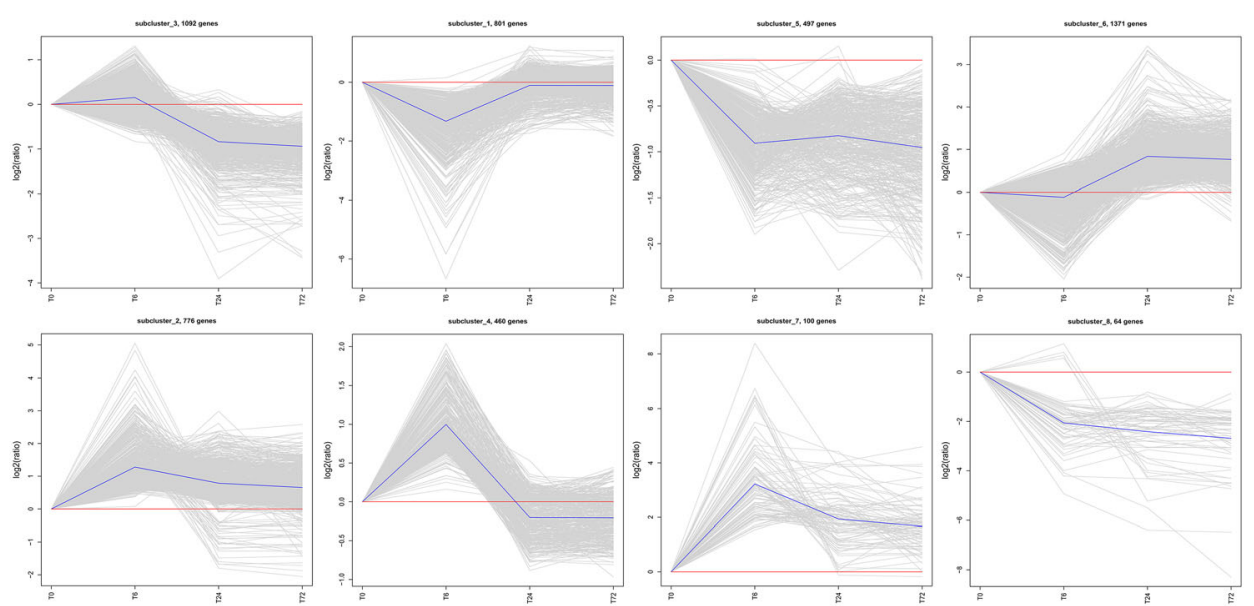

Fig. 3 . a Hierarchical cluster analysis, using the FPKM value of the differential gene as the expression level; the areas with different colours represent different clustering grouping information. b Differential genetic clustering of the H-cluster method.

\section{GO and KEGG analysis of differential genes}

GO and KEGG pathway enrichment analyses were conducted for the differential genes. Significant enrichment was denoted when FDR $\leq 0.05$. The results found a lack of significant enrichment in T6 vs. T0. However, 17 functional groups were significantly enriched in $\mathrm{T} 24$ vs. T0, and the highest proportion of "organonitrogen compounds" in the biological processes class was $16.93 \%$. In $\mathrm{T} 72$ vs. T0, a total of 62 functional groups were significantly enriched. The highest proportion of "cellular metabolic processes" in the biological processes class was $19.07 \%$, the highest proportion of "thylakoids" in the cell composition class was $16.22 \%$, and the highest proportion of "small molecule binding" in the molecular function class was $6.10 \%$ (Fig. S1 and Fig. S2). The analysis showed that up-regulated transcripts in biological processes primarily derived from the pathways of "phosphate-containing compound metabolic process", "protein metabolic process", "protein modification process", and "macromolecule modification". In addition, up-regulated transcripts in cell composition were mostly related to photosynthesis, including "photosystem", "photosynthetic membrane", and "thylakoid". In terms of molecular function, many up-regulated transcripts were related to enzyme activity, including "kinase activity", "transferase activity", "protein kinase activity", and "phosphotransferase activity", whilst others were related to substance synthesis, including "ATP 
T6 VS T0

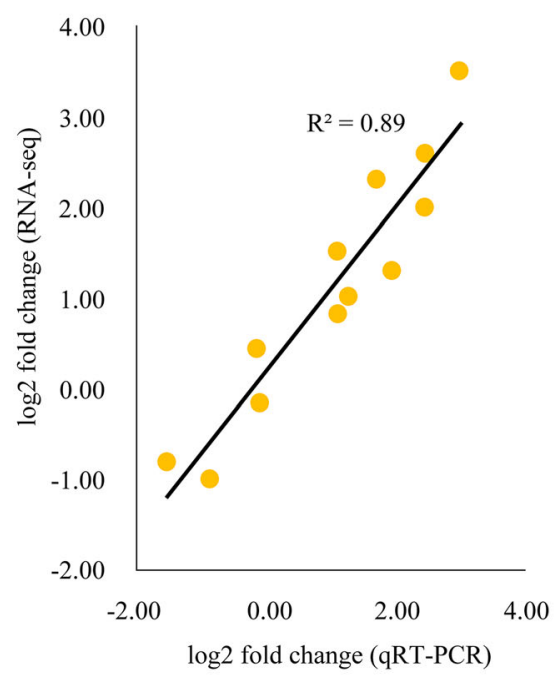

T24 VS T0

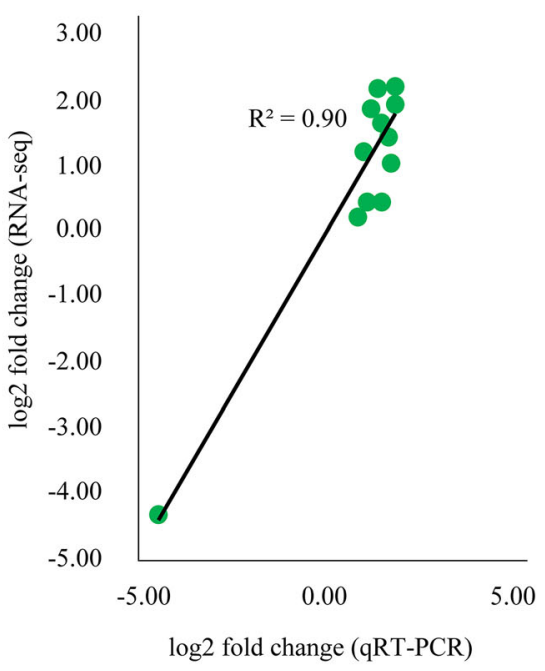

T72 VS T0

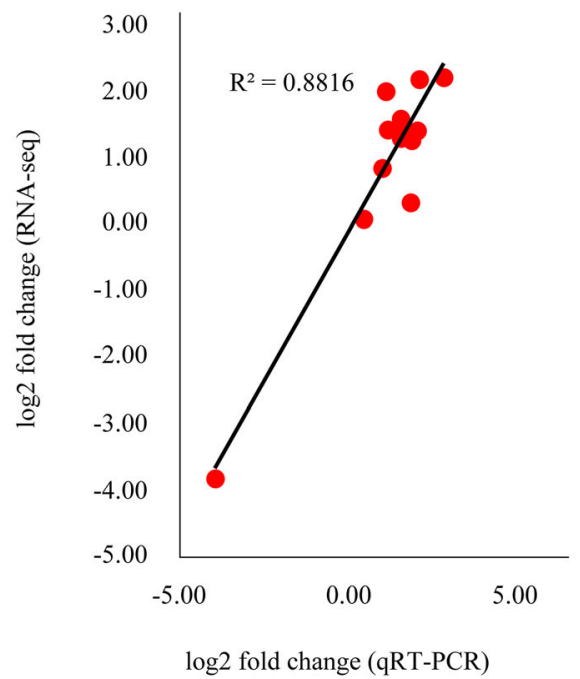

Fig. 4 Correlation between RNA-seq and qRT-PCR results. Twelve genes were randomly selected to verify the transcriptome sequencing results

binding", "small molecule binding", "nucleotide binding", and "anion binding".

The result of KEGG enrichment analysis showed 1150 differential genes belonging to 113 KEGG pathways in the T6 vs. T0 group, but a significant enrichment pathway was not identified. The T24 vs. T0 group had 1138 differential genes belonging to 106 KEGG pathways, of which the "photosynthesis-antenna protein" pathway was significantly enriched. Furthermore, the T72 vs. T0 group showed 1553 differential genes belonging to 116 KEGG pathways, and the "photosynthesis-antenna protein" pathway was also significantly enriched at $72 \mathrm{~h}$ (Fig. S3).

\section{Salt response-related transcription factor}

A total of 362 transcripts were identified from the 4 transcripts (T0, T6, T24, and T72), which belonged to 56 gene families. Of these families, 13 had more than 10 genes
(Fig. 5a). In the T6 vs. T0 group, differentially expressed transcription factors belonged to 29 families. The gene families with more than 5 genes included the basic helixloop-helix (bHLH) gene family (11 entries), WRKY gene family (8 entries), MYB gene family ( 7 entries), and ethylene response factor (ERF) (7 entries). In the T24 vs. T0 group, differentially expressed transcripts belonged to 25 families; those that comprised more than 5 genes included the ERF gene family (17 entries), WRKY gene family (14 entries), bHLH gene family (8 entries), and the MYB gene family (7 entries). The T72 vs. T0 group had differentially expressed transcripts belonging to 31 gene families, and those that included more than 5 genes were the WRKY gene family (18 entries), bHLH gene family (11 entries), ERF (10 entries), DIVARICATA gene family (6 entries), and MYB gene family (5 entries). The T6 vs. T0, T24 vs. T0, and T72 vs. T0 ranges co-expressed transcripts belonging to 20 gene families, which are
A

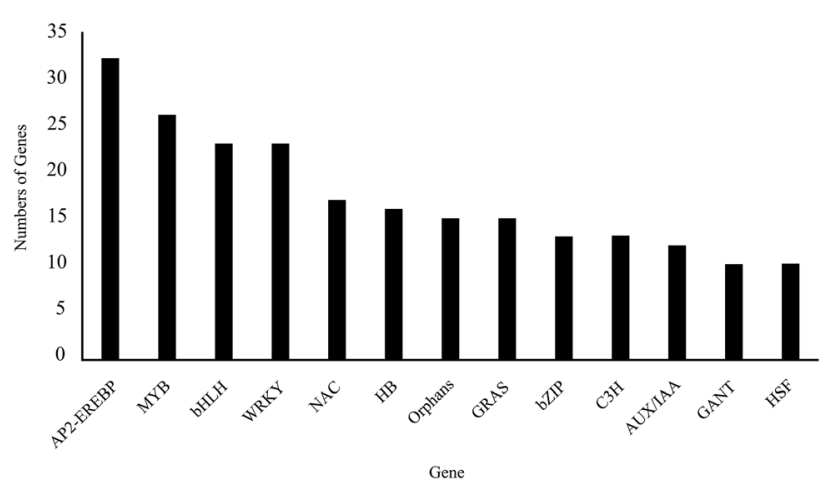

B

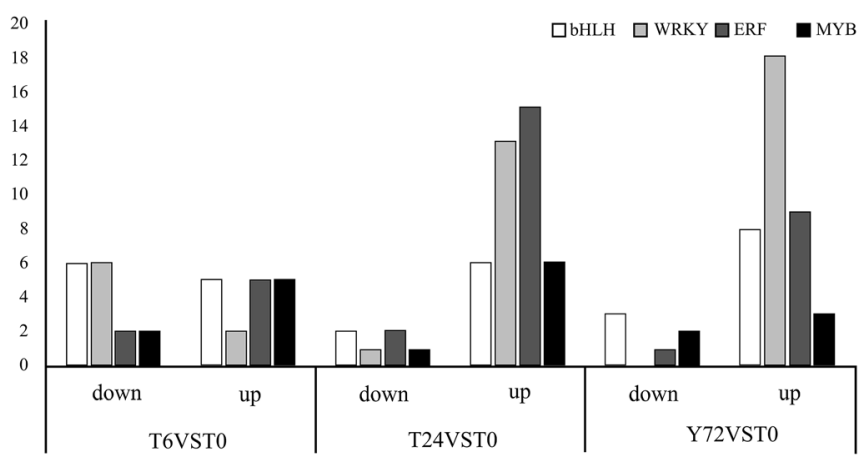

Fig. 5 a 13 gene families with more than 10 transcription factors. b Differential expression of four transcription factor families under salt stress 
possibly related to salt tolerance. Among these families, bHLH, WRPK, ERF, and MYB included the largest number of genes. Four of the gene families presented gene expression at $6 \mathrm{~h}, 24 \mathrm{~h}$, and $72 \mathrm{~h}$ of stress (Fig. 5b). Moreover, the transcription factors up-regulated at $24 \mathrm{~h}$ and $72 \mathrm{~h}$ stress were more than that at $6 \mathrm{~h}$ stress, with the exception of the MYB gene family whereby the up-regulated genes at $72 \mathrm{~h}$ were lower than $6 \mathrm{~h}$. However, the transcription factors down-regulated at $6 \mathrm{~h}$ of stress were more than that at $24 \mathrm{~h}$ and $72 \mathrm{~h}$.

\section{Identification of DEGs in different treatment periods}

The genes that showed a statistically significant difference in expression levels at T6, T24, or T72, compared with those at $\mathrm{T} 0$, were enriched to reveal the specific genes and their functions that were significantly different in the early stage of salt tolerance in $P$. mume. The functional categories of the differentially expressed genes (DEGs) significantly up-regulated are shown in Fig. 6a. The results showed that a large number of transcription factors and hormone signal transduction genes were up-regulated at T6, T24, and T72. Sucrase synthase (SUSY) and photosystem I reaction centre (PS I) subunit genes of the metabolic signal transduction pathway were significantly upregulated, indicating their considerable role in signal transduction in the early stage of salt tolerance. Several genes related to ion transport and reconstruction of ion balance, as well as genes related to the biosynthesis of osmotic protective substances, reactive oxygen species scavenging, and stress resistance-related protein genes were also significantly up-regulated. The functional categories of DEGs with significantly down-regulated expression are shown in Fig. 6b. Numerous transcription factors and aquaporins (AQPs) related to dehydration protection and water transport were significantly down-regulated in T6, T24, and T72. These findings suggest that regulatory networks involving hormone signal transduction, ion transport and reconstruction of ion balance-related genes, biosynthesis of osmotic protective substances-, reactive oxygen species scavenging, and stress resistance-related genes regulated the early salt tolerance activities of $P$. mume.

Based on the enrichment of GO and KEGG, proteinprotein interaction analysis of DEGs and TFs was undertaken to identify the key genes conveying salt tolerance in $P$. mume. It was possible that these structural genes were regulated by bZIP family transcription factors. In addition, cyclic-nucleotide gated channels (CNGCs), POD, and SUSY may contribute to salt tolerance of $P$. mume (Fig. 7).

\section{Discussion}

\section{Genes related to ion transport and reconstruction of ion balance}

Plants must undergo osmotic adjustments to rebuild the ion balance when under certain environmental adversity, like
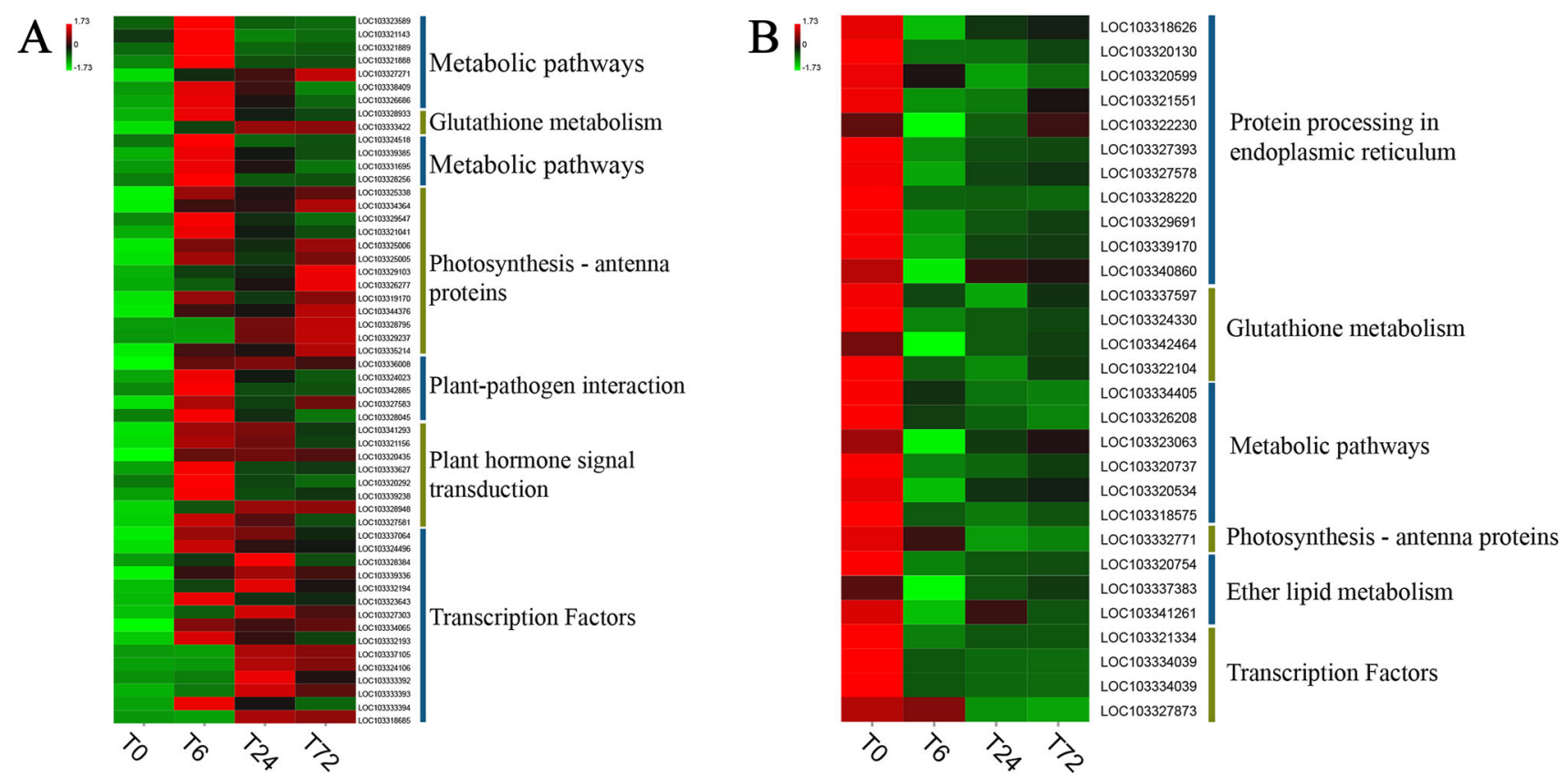

Fig.6 Heatmap comparison of a significantly up-regulated DEGs and b significantly down-regulated DEG, in three treatment periods. The functional categories of significantly over-represented DEGs were identified in T0 and T72 


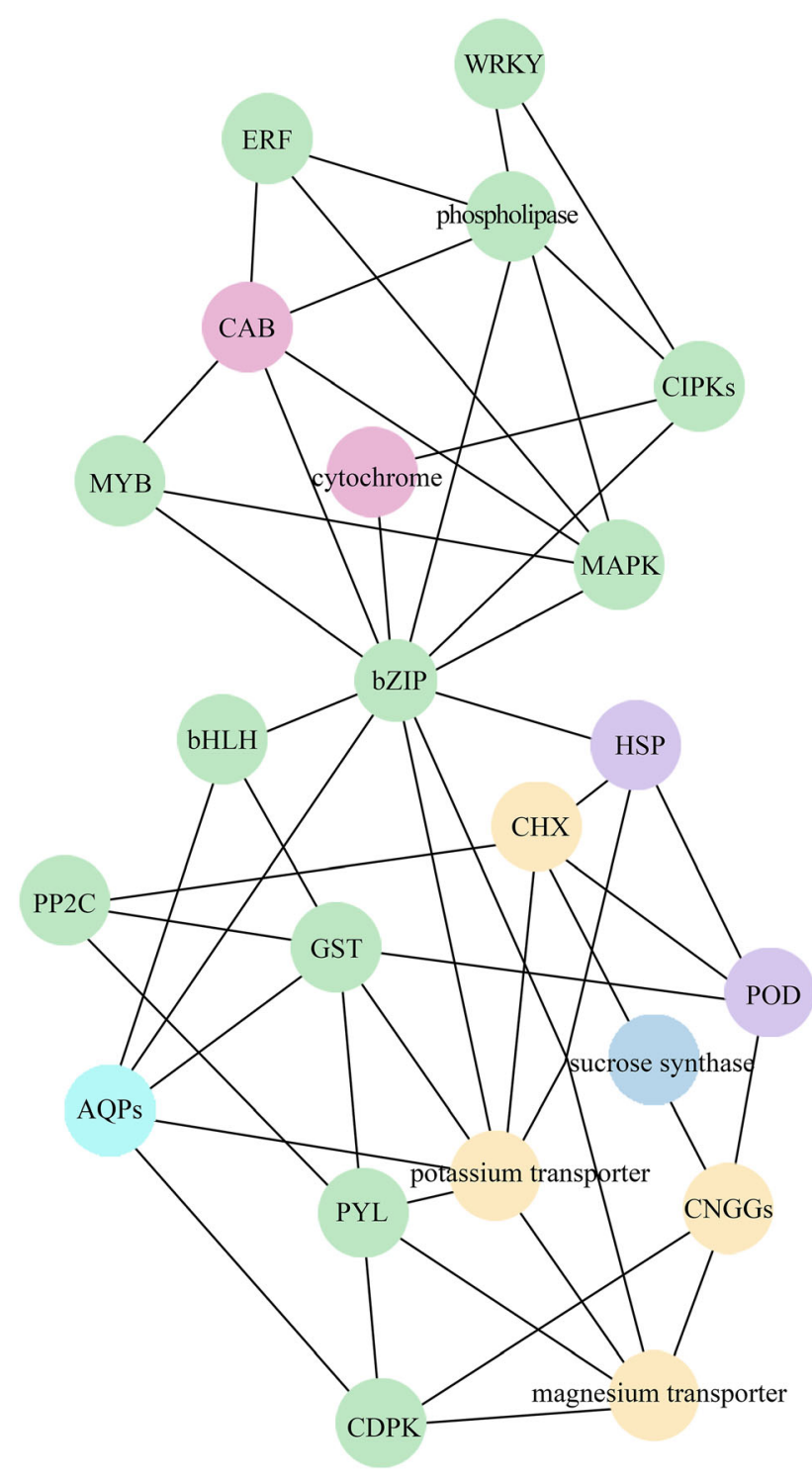

Fig.7 Construction of protein-protein interaction network model of salt-tolerant candidate genes in $P$. mume using Cytoscape software

salt stress. Many genes were found to be involved in the reconstruction of ion balance in the gene expression profile of $P$. mume. The $\mathrm{K}^{+}$ion channel (AKT) was up-regulated at $24 \mathrm{~h}$ of salt stress, while the $\mathrm{Mg}^{2+}$ transporter was upregulated at $6 \mathrm{~h}, 24 \mathrm{~h}$, and $72 \mathrm{~h}$ of stress. In addition, the $\mathrm{K}^{+}$transporter was also up-regulated at $6 \mathrm{~h}, 24 \mathrm{~h}$, and $72 \mathrm{~h}$ (Table 3a). Previous studies have shown that cation/ $\mathrm{H}+$ exchangers (CHX) may also participate in sodium efflux (Manohar et al. 2011). In the present study, a CHX18 gene was found to be up-regulated at $6 \mathrm{~h}$ and $72 \mathrm{~h}$ in the differential expression profile of $P$. mume, which may be involved in ion metabolism regulated by CHX. A comparison of the differential expression profiles of salt stress at $6 \mathrm{~h}, 24 \mathrm{~h}$, and $72 \mathrm{~h}$ identified greater down-regulation of genes than up-regulation in the genes corresponding to ion balance at $6 \mathrm{~h}$ stress, whilst greater up-regulation than down-regulation was established of the genes at $24 \mathrm{~h}$ and $72 \mathrm{~h}$ of stress. Ion metabolism and transport are significant areas in relation to the salt tolerance of $P$. mume because salt stress affects the ion balance, and the plant uses the process of ion transport to regain such balance.

\section{Genes related to biosynthesis of osmotic protective substances}

The differential expression profile of $P$. mume showed the up-regulation of SUSY genes after stress. Sugars, polyols, amino acids (such as Pro), and betaine are all non-toxic soluble substances, which accumulate in the cytoplasm in an external hypertonic environment and have no effect on cellular metabolism (Chen et al. 2002). Pyrroline-5-carboxylate synthase (P5CS) is a key enzyme for Pro synthesis and degradation; up-regulation of the P5CS gene was identified at $6 \mathrm{~h}$ of stress. The P5CS gene has been successfully overexpressed in many plants such as arabidopsis and tobacco, and can effectively improve the salt tolerance of transgenic plants (Cao et al. 2011; Huang et al. 2013). The differential expression profile showed only one mannitol dehydrogenase gene, which was down-regulated at $6 \mathrm{~h}$ and $24 \mathrm{~h}$ of stress (Table 3b). Subsequent data analysis found that most of the key genes encoding osmotic protective substances lacked significant expression changes. It is possible that these genes had not yet accumulated during the stress response process at the selected time ranges, and that they primarily assist the process of stress resistance.

\section{Genes related to active reactive oxygen species scavenging and anti-stress proteins}

Under salt stress, plant cells produce large amounts of reactive oxygen species due to hindered metabolism. These species include oxygen free radicals $\left(\mathrm{O}^{2-} \cdot\right.$ ), hydrogen peroxide $\left(\mathrm{H}_{2} \mathrm{O}_{2}\right)$, and hydroxyl free radicals $(\cdot \mathrm{OH})$. Although a normal product of cellular reactions, an excessive amount of reactive oxygen species is harmful to cells as they can cause lipid peroxidation of cell membranes and DNA damage that leads to cell death (Luo et al. 2020). To overcome this potential issue, plants synthesise antioxidants, which are renowned for their free radical scavenging ability. Many antioxidant genes, such as POD, SOD, metallothionein protein (MT), glutathione sulfur transferase, aldehyde dehydrogenase (ALDH), and peroxiredoxin (PrxR), were detected in the differential expression profile of $P$. mume (Table 3c). In high-salt and arid environments, the ALDH gene family was up-regulated, indicating their correlation to the stress response in cotton (Guo et al. 2017). Data analysis of the antioxidant genes identified greater up-regulation of POD, MT, glutathione 
Table 3 Key genes conveying salt tolerance in P. mume

\begin{tabular}{|c|c|c|c|c|c|c|}
\hline \multirow[t]{2}{*}{ Genes } & \multicolumn{2}{|l|}{ T6VST0 } & \multicolumn{2}{|l|}{ T24VST0 } & \multicolumn{2}{|l|}{ T72VST0 } \\
\hline & Down -regulated & Up-regulated & Down-regulated & Up-regulated & Down -regulated & Up -regulated \\
\hline
\end{tabular}

a. Genes related to ion transport and reconstruction of ion balance

$\begin{array}{ll}\text { V-ATPase } & 0 \\ \text { phospholipid-transporting ATPase } & 0 \\ \text { plasma membrane ATPase } & 1 \\ \text { NHX } & 3 \\ \text { AKT } & 1 \\ \text { potassium transporter } & 2 \\ \text { magnesium transporter } & 0 \\ \text { CHX } & 1 \\ \text { CNGCs } & 0\end{array}$

$\begin{array}{ll}1 & 0 \\ 0 & 0 \\ 0 & 0 \\ 0 & 0 \\ 0 & 0 \\ 2 & 0 \\ 1 & 0 \\ 1 & 1 \\ 2 & 0\end{array}$

b. Genes related to biosynthesis of osmotic protective substances

$\begin{array}{lll}\text { P5CS } & 0 & 1 \\ \text { sucrose synthase } & 0 & 1 \\ \text { mannitol dehydrogenase } & 1 & 0\end{array}$

c. Genes related to active oxygen scavenging and anti-stress proteins

\begin{tabular}{|c|c|c|c|c|c|}
\hline POD & 5 & 4 & 0 & 3 & 1 \\
\hline SOD & 0 & 0 & 3 & 0 & 3 \\
\hline MT & 0 & 0 & 0 & 2 & 0 \\
\hline Glutathione S-transferase & 3 & 1 & 4 & 2 & 2 \\
\hline ALDH & 0 & 2 & 0 & 1 & 0 \\
\hline PrxR & 0 & 0 & 3 & 0 & 2 \\
\hline HSP & 18 & 1 & 18 & 1 & 16 \\
\hline disease resistance protein & 6 & 7 & 0 & 13 & 1 \\
\hline
\end{tabular}

d. Genes related to dehydration protection and water transport

$\begin{array}{lll}\text { AQPs } & 5 & 1 \\ \text { LEA } & 0 & 0 \\ \text { DHN } & 0 & 0\end{array}$

e. Genes related to stress signal transduction

\begin{tabular}{|c|c|c|c|c|c|c|}
\hline MAPK & 5 & 2 & 0 & 3 & 0 & 8 \\
\hline CDPK & 0 & 3 & 0 & 3 & 0 & 5 \\
\hline phospholipase & 2 & 3 & 1 & 2 & 3 & 2 \\
\hline CIPKs & 7 & 1 & 0 & 2 & 1 & 2 \\
\hline CBLs & 1 & 0 & 0 & 0 & 0 & 4 \\
\hline CAMTAs & 0 & 0 & 0 & 1 & 0 & 1 \\
\hline $\mathrm{PP} 2 \mathrm{C}$ & 7 & 4 & 3 & 7 & 0 & 9 \\
\hline PYL & 0 & 1 & 0 & 1 & 0 & 1 \\
\hline PYR & 0 & 0 & 0 & 0 & 0 & 1 \\
\hline SnRKs & 0 & 0 & 0 & 1 & 0 & 1 \\
\hline bZIP & 1 & 1 & 1 & 1 & 1 & 1 \\
\hline MYB & 2 & 5 & 1 & 6 & 2 & 3 \\
\hline MYC & 1 & 0 & 0 & 1 & 1 & 0 \\
\hline
\end{tabular}

sulfur transferase, and ALDH than down-regulation as the stress time increased;
In addition, regarding physiological response, SOD enzyme activity and expression of the SOD gene decreased after $24 \mathrm{~h}$ of stress, which implies that SOD was not the 
key gene involved in salt tolerance of the $P$. mume in this study. This finding differs from previous results studying the salt stress tolerance of barley and arabidopsis, whereby the SOD gene was depicted as being highly involved in salt tolerance (Zhang et al. 2021; Zheleznova et al. 2020). This difference may be due to differences in salinity tolerance of species or treatment conditions. Therefore, the role of the SOD gene in the salt stress tolerance of $P$. mume needs further exploration and verification.

\section{Genes related to dehydration protection and water transport}

Two AQP genes were up-regulated after stress exposure, while 9 AQP genes were shown to be down-regulated after stress. The results of the study established an increase in the expression of LEA and DHN genes of P. mume at $24 \mathrm{~h}$ and $72 \mathrm{~h}$ of stress. LEA proteins possess high hydrophilicity and thermal stability, which enables stablisation of the cell membrane structure, and can be used as molecular chaperones. Furthermore, LEA can be used to bind metal ions and prevent oxidation in cells, and induce expression under abiotic stresses including salt, drought, and cold temperatures (Cheng et al. 2021). DHN is a member of the LEA family; studies have found that overexpression of the DHN gene can enhance the response of Arabidopsis seeds to salt stress (Williams et al. 2015). In terms of P. mume, Du et al. (2012) identified 6members of the DHN gene family in the P. mume genome. With the exception of PmLEA29, the expression levels of the remaining 5 DHN genes increased under salt stress (Table 3d). Therefore, dehydration protection is an important approach for salt tolerance of $P$. mume.

\section{Genes related to stress signal transduction}

In the calcineurin $\mathrm{B}$-like protein-CBL-interacting protein kinase (CBL-CIPK) pathway, the CBL, CIPK, and calmodulin-binding transcription activator (CAMTA) gene families all showed changes in their expression levels. CBLs and CIPKs jointly participate in the process of ion stress signal transduction. Low $\mathrm{K}^{+}$content and high $\mathrm{Mg}^{2+}$ content generates an ion stress signal, which subsequently causes the interaction of transmembrane proteins with their respective target proteins through $\mathrm{Ca}^{2+}$ flow. This initiates cascade phosphorylation (CBLs and CIPKs) (Boudsocq et al. 2013), which results in corresponding changes to the transcription factors of downstream related genes. Alternatively, cascade phosphorylation results in direct interaction with $\mathrm{Ca}^{2+}$ transcription activators, such as CAMTAs and GT element-binding-like proteins (GTLs) (Pandey et al. 2013; Hua et al. 2012) to regulate the salt stress ionic balance genes and the expression of transcription factors related to stress resistance.

In the signal transduction pathways of mitogen-activated protein kinase (MAPK) and calcium-dependent protein kinase (CDPK), these protein kinases were up-regulated at $6 \mathrm{~h}, 24 \mathrm{~h}$, and $72 \mathrm{~h}$ of stress. It could be speculated that the MAPK cascade achieves osmotic balance by adjusting the osmotic solute, including the functionally interconnected protein kinases of MAPK, MAPK kinase (MAPKK), and MAPK kinase kinase (Table 3e). In the differential expression profile of $P$. mume, only changes in the expression of phospholipase were detected; yet the corresponding gene of its hydrolysate was not found. Overall, establishing whether salt stress initiates the phospholipase transduction pathway requires more in-depth research.

Analysis of the DEGs of P. mume showed more downregulated genes of PP2C than up-regulated genes at $6 \mathrm{~h}$ of stress, whilst more up-regulated genes were detected at $24 \mathrm{~h}$ and $72 \mathrm{~h}$ than down-regulated genes. The gene expression of PYL, PYR, and SnRKs increased post-stress, which subsequently positively regulated the abscisic acid (ABA) signalling pathway (Soon et al. 2012). In pathways involving protein synthesis, salt stress signals were recognised by $\mathrm{ABA}$ and were then transmitted to downstream transcription factors, such as MYB and MYC. These intermediate proteins recognise each other due to cis-regulatory elements, like MYBRS and MYCRS which are drought responsive elements, on the promoters of osmotic stress-induced genes. This enables the regulation of gene expression in response to salt stress (Yong et al. 2019; Li et al. 2010). The expression of bZIP, MYB, and MYC transcription factors in $P$. mume varies significantly; however, these factors all participate in the ABA-dependent signal transduction pathway.

\section{Analysis of the internal mechanism of physiological response}

The internal mechanism of the physiological response of $P$. mume to salt stress was analysed according to the physiological response results alongside the expression of key genes (Table 3). In the early stage of stress, the photosynthetic gas exchange parameters of Pn, Gs, and Tr all decreased, while $\mathrm{Ci}$ increased; the initial decline of Pn to $\mathrm{Ci}$ reflects the efficiency of carbon carboxylation, and correlation with RuBisCO activity was identified to an extent (Nik et al. 2020). Two RuBisCO-related genes were identified in the differential gene spectrum of P. mume; these were down-regulated at $24 \mathrm{~h}$ and $72 \mathrm{~h}$ of salt stress, which was consistent with the physiological results, as salt stress inhibits the activity of Rubisco, which is the key photosynthetic enzyme of $P$. mume. Consequently, the photosynthetic rate was found to decrease. In addition, 
P5CS and SUSY genes related to Pro synthesis were detected in the gene expression profile of $P$. mume; these genes regulate the synthesis of Pro and sucrose, promote the accumulation of Pro and soluble sugar under salt stress, and alleviate osmotic stress (Funck et al. 2020).

The results also portrayed the important role played by POD in the removal of reactive oxygen species from plant cells, and the regulation of POD enzyme synthesis by POD genes. POD enzyme activity increased after 10 days of stress. In terms of transcription level, POD genes were upregulated at $6 \mathrm{~h}$ and $24 \mathrm{~h}$ of stress, and up-regulated genes were more than down-regulated genes. Therefore, gene expression can be viewed as related to protein synthesis, but not completely unified.

Acknowledgements This wok was supported by the State Forestry Administration of China (2017-LY-039). Thanks to the teachers and the foundation for their support.

Authors contribution All authors contributed to the study conception and design. Material preparation, data collection, and analysis were performed by Qingwei Li. The first draft of the manuscript was written by Xiujun Wang and Zhenqi Song; all authors commented on previous versions of the manuscript. All authors read and approved the final manuscript.

\section{Declarations}

Conflict of interest All authors have read and approved this version of the article, and due care has been taken to ensure the integrity of the work. No conflict of interest exists in the submission of this manuscript.

Open Access This article is licensed under a Creative Commons Attribution 4.0 International License, which permits use, sharing, adaptation, distribution and reproduction in any medium or format, as long as you give appropriate credit to the original author(s) and the source, provide a link to the Creative Commons licence, and indicate if changes were made. The images or other third party material in this article are included in the article's Creative Commons licence, unless indicated otherwise in a credit line to the material. If material is not included in the article's Creative Commons licence and your intended use is not permitted by statutory regulation or exceeds the permitted use, you will need to obtain permission directly from the copyright holder. To view a copy of this licence, visit http://creativecommons. org/licenses/by/4.0/.

\section{References}

Agarwal P, Khurana P (2020) TaZnF, a C3HC4 type RING zinc finger protein from Triticum aestivum is involved in dehydration and salinity stress. J Plant Biochem Biot 29:395-406

Bao F, Du D, An Y, Yang W, Wang J, Cheng T, Zhang Q (2017) Overexpression of Prunus mume Dehydrin Genes in Tobacco Enhances Tolerance to Cold and Drought. Front Plant Sci 8.

Boudsocq M, Sheen J (2013) CDPKs in immune and stress signaling. Trends Plant Sci 18:30-40
Calestani C, Moses MS, Maestri E, Marmiroli N, Bray EA (2015) Constitutive expression of the barley dehydrin gene aba2 enhances Arabidopsis germination in response to salt stress. Int J Plant Biol 6(1):1039-1054

Cao L, Yi MF, Sun ZY, Han L, Ju GS, Ma XR (2011) Site directed mutagenesis of the LpP5CS gene of Lolium perenne and its transformation in Arabidopsis thaliana. Acta Pratacul Sin 20(1):242-247

Chen P, Zhao X, Tian GG, Yuan X, Li X, Li Z, Yu X, Hu R, Wang Y, Pei $X$ et al (2021) C28 induced autophagy of female germline stem cells in vitro with changes of H3K27 acetylation and transcriptomics. Gene 766:145-150

Chen THH, Murata N (2002) Enhancement of tolerance of abiotic stress by metabolic engineering of betaines and other compatible solutes. Curr Opin Plant Biol 5:250-257

Cheng Z, Zhang X, Yao W, Zhao K, Liu L, Fan G, Zhou B, Jiang T (2021) Genome-wide search and structural and functional analyses for late embryogenesis-abundant (LEA) gene family in poplar. Bmc Plant Biol 21(1101).

Du D, Zhang Q, Cheng T, Pan H, Yang W, Sun L (2012) Genomewide identification and analysis of late embryogenesis abundant (LEA) genes in Prunus mume. Mol Biol Rep 40:1937-1946

Funck D, Baumgarten L, Stift M, von Wiren N, Schoenemann L (2020) Differential Contribution of P5CS Isoforms to Stress Tolerance in Arabidopsis. Front Plant Sci 11(565134).

Shi GY, Wang YM, Hua JP (2012a) Aquaporins and Salt Tolerance of Higher Plants. J Agr Sci Tec 14(4):31-38

Goetz K, Naher J, Fettke J, Chmielewski F (2018) Changes of proteins during dormancy and bud development of sweet cherry (Prunus avium L.). Sci Hortic-Amsterdam 239:41-49

Guo X, Wang Y, Lu H, Cai X, Wang X, Zhou Z, Wang C, Wang Y, Zhang Z, Wang K et al (2017) Genome-wide characterization and expression analysis of the aldehyde dehydrogenase (ALDH) gene superfamily under abiotic stresses in cotton. Gene 628:230-245

Huang C, Jiang W, Yang L, Li Y, Yang C (2013) Response of antisense ScP5CS transgenic tobacco plants to PEG and $\mathrm{NaCl}$ treatments. Crops 3(2013):42-45

Kanehisa M, Araki M, Goto S, Hattori M, Hirakawa M, Itoh M, Katayama T, Kawashima S, Okuda S, Tokimatsu T et al (2008) KEGG for linking genomes to life and the environment. Nucleic Acids Res 36:D480-D484

Li C, Lv J, Zhao X, Ai X, Zhu X, Wang M, Zhao S, Xia G (2010) TaCHP: a wheat zinc finger protein gene down-regulated by abscisic acid and salinity stress plays a positive role in stress tolerance. Plant Physiol 154:211-221

Li HS (2003) Principles and techniques of plant physiology and biochemistry experiment. Higher Education Press, Beijing, China

Li QW (2009) Studies on Investigation of Wild Mei Germplasm Resources of Sichan Yunnan Tibet and Guizhou Province as well as Regional Tests of Hardy Mei Cultivars in North China. Beijing Forestry University.

Luo Z, Szczepanek A, Abdel-Haleem H (2020) Genome-Wide Association Study (GWAS) Analysis of Camelina Seedling Germination under Salt Stress Condition. Agronomy-Basel 10(14449).

Manohar M, Shigaki T, Hirschi KD (2011) Plant cation $/ \mathrm{H}^{+}$ exchangers (CAXs): biological functions and genetic manipulations. Plant Biol 13:561-569

Nik Muhammad Nasir NN, Khandaker MM, Mohd KS, Badaluddin NA, Osman N, Mat N (2020) Effect of Hydrogen Peroxide on Plant Growth, Photosynthesis, Leaf Histology and Rubisco Gene Expression of theFicus deltoideaJack Var.deltoideaJack. J Plant Growth Regul. 
Pandey N, Ranjan A, Pant P, Tripathi RK, Ateek F, Pandey HP, Patre UV, Sawant SV (2013) CAMTA 1 regulates drought responses in Arabidopsis thaliana. Bmc Genomics 14.

Peng T, Guo C, Yang J, Xu M, Zuo J, Bao M, Zhang J (2016) Overexpression of a Mei (Prunus mume) CBF gene confers tolerance to freezing and oxidative stress in Arabidopsis. Plant Cell Tiss Org 126:373-385

Perez-Rodriguez P, Riano-Pachon DM, Correa LGG, Rensing SA, Kersten B, Mueller-Roeber B (2010) PInTFDB: updated content and new features of the plant transcription factor database. Nucleic Acids Res 381:D822-D827

Przedpelska-Wasowicz EM, Wierzbicka M (2011) Gating of aquaporins by heavy metals in Allium cepa L. epidermal cells. Protoplasma 248:663-667

Shi GY, Wang YM, Hua JP (2012b) Aquaporins and Salt Tolerance of Higher Plants. J Agric Sci Technol 14:31-38

Soon F, Ng L, Zhou XE, West GM, Kovach A, Tan MHE, SuinoPowell KM, He Y, Xu Y, Chalmers MJ et al (2012) Molecular mimicry regulates ABA signaling by SnRK2 kinases and PP2C phosphatases. Science 335:85-88

Sun X, Sun C, Li Z, Hu Q, Han L, Luo H (2016) AsHSP17, a creeping bentgrass small heat shock protein modulates plant photosynthesis and ABA-dependent and independent signalling to attenuate plant response to abiotic stress. Plant Cell Environ 39:1320-1337

Wan X, Yang J, Li X, Zhou Q, Guo C, Bao M, Zhang J (2016) Overexpression of PmHSP17.9 in Transgenic Arabidopsis thaliana confers Thermotolerance. Plant Mol Biol Rep 34:899-908

Wang L, Zhang C, Wang Y, Wang Y, Yang C, Lu M, Wang C (2018) Tamarix hispida aquaporin ThPIP2;5 confers salt and osmotic stress tolerance to transgenic Tamarix and Arabidopsis. Environ Exp Bot 152:158-166

Wang XK, Huang JL (2015) Principles and techniques of plant physiology and biochemistry experiment. Higher Education Press, Beijing, China

Waqas M, Chen Y, Iqbal H, Shareef M, Rehman HU, Bilal HM (2021) Synergistic consequences of salinity and potassium deficiency in quinoa: linking with stomatal patterning, ionic relations and oxidative metabolism. Plant Physiol Bioch 159:17-27

Weng H, Yoo CY, Gosney MJ, Hasegawa PM, Mickelbart MV (2012) Poplar GTL1 Is a Ca2+/Calmodulin-Binding Transcription
Factor that Functions in Plant Water Use Efficiency and Drought Tolerance. Plos One 7(3)

Yang J, Wan XL, Guo C, Zhang JW, Bao MZ (2016) Identification and expression analysis of nuclear factor Y families in Prunus mume under different abiotic stresses. Biol Plantarum 60:419-426

Yang JX, Li QW, Liu YX, Xu XL, Zhang QX, Li WG, Sun YF (2018) Effects of $\mathrm{NaCl}$ Stress on the Germination and Physiological Characteristics of Prunus mume and Prunus davidiana Seeds. J Northwest Forestry Univ 33(2):94-99

Yong Y, Zhang Y, Lyu Y (2019) A MYB-Related Transcription Factor from Lilium lancifolium L. (LlMYB3) Is Involved in Anthocyanin Biosynthesis Pathway and Enhances Multiple Abiotic Stress Tolerance in Arabidopsis thaliana. Int J Mol Sci 20(319513).

Young MD, Wakefield MJ, Smyth GK, Oshlack A (2010) Gene ontology analysis for RNA-seq: accounting for selection bias. Genome Biol 11(2).

Zhai M, Sun Y, Jia C, Peng S, Liu Z, Yang G (2016) Over-expression of JrsHSP17.3 gene from Juglans regia confer the tolerance to abnormal temperature and $\mathrm{NaCl}$ stresses. J Plant Biol 59:549-558

Zhang Q, Chen W, Sun L, Zhao F, Huang B, Yang W, Tao Y, Wang J, Yuan Z, Fan Get al (2012) The genome of Prunus mume. Nat Commun 3

Zhang X, Zhang L, Chen Y, Wang S, Fang Y, Zhang X, Wu Y, Xue D (2021) Genome-wide identification of the SOD gene family and expression analysis under drought and salt stress in barley. Plant Growth Regul

Zhao W, Faust F, Schubert S (2020) Potassium is a potential toxicant for Arabidopsis thaliana under saline conditions. J Plant Nutr Soil Sc 183:455-467

Zheleznova N, Terashvili M, Bullis J, Stumpf M, Linh N, Geurts A, Lombard J (2020) Blood Pressure, Vascular Reactivity, and SOD Expression/Activity in Mas1 Receptor Knockout Rats in the Dahl Salt-Sensitive Genetic Background. Faseb J 341

Zhu J (2016) Abiotic Stress Signaling and Responses in Plants. Cell $167: 313-324$

Publisher's Note Springer Nature remains neutral with regard to jurisdictional claims in published maps and institutional affiliations. 